\title{
A Hemodynamic Mechanism Correlating with the Initiation of MCA Bifurcation Aneurysms
}

\author{
(D) Z. Huang, (D) M. Zeng, (D)W.G. Tao, DF.Y. Zeng, (D)C.Q. Chen, (D) L.B. Zhang, and DF.H. Chen
}

O-

\begin{abstract}
BACKGROUND AND PURPOSE: Previous studies have reported that MCA bifurcation aneurysms usually emerge on inclined bifurcations; however, the reason is unclear. We designed this study to explore hemodynamic mechanisms that correlate with the initiation of MCA bifurcation aneurysms.
\end{abstract}

MATERIALS AND METHODS: Fifty-four patients with unilateral MCA bifurcation aneurysms and 54 control patients were enrolled in this study after propensity score matching, and their clinical and CTA data were collected. We extracted the morphologic features of aneurysmal MCA bifurcations to build a simplified MCA bifurcation model and performed a computational fluid dynamics analysis.

RESULTS: The presence of MCA aneurysms correlated with smaller parent-daughter angles of MCA bifurcations $(P<.001)$. Aneurysmal MCA bifurcations usually presented with inclined shapes. The computational fluid dynamics analysis demonstrated that when arterial bifurcations became inclined, the high-pressure regions and low wall shear stress regions shifted from the apexes of the arterial bifurcations to the inclined daughter arteries, while the initial sites of MCA bifurcation aneurysms often overlapped with the shifted high-pressure regions and low wall shear stress regions.

CONCLUSIONS: Our results suggest that the initiation of MCA bifurcation aneurysms may correlate with shifts of high-pressure regions and low wall shear stress regions that occur on inclined MCA bifurcations.

ABBREVIATIONS: CFD = computational fluid dynamics; HPR = high-pressure region; LWSS = low wall shear stress; LWSSR = low wall shear stress region; $\mathrm{PSM}=$ propensity score matching; $\mathrm{RD}=$ ratio of diameter; $\mathrm{ROC}=$ receiver operating characteristic

$\mathbf{T}$ he occurrence of intracranial aneurysms is generally thought to be due to arterial wall weakening and/or the influence of hemodynamics. ${ }^{1-4}$ Most studies on aneurysm etiology have focused on hemodynamics. Because the geometric shapes of intracranial arteries are diverse, their hemodynamics are also complex. Arterial bifurcations are common sites for intracranial aneurysms. The bifurcations are generally exposed to extreme hemodynamic stress; those with special morphologic features that significantly

Received November 29, 2019; accepted after revision February 22, 2020

From the Departments of Neurosurgery (Z.H., M.Z., F.H.C., W.G.T.), and Radiology (F.Y.Z., C.Q.C.), Xiangya Hospital, Central South University, Changsha, China; and Department of Neurosurgery (L.B.Z.), School of Medicine, Yale University, New Haven, Connecticut.

This work was supported by National Natural Science Foundation of China (grant No. 81873756)

Please address correspondence to Fenghua H. Chen, PhD, MD, Department of Neurosurgery, Xiangya Hospital, Central South University, No. 87 Xiangya Rd, Kaifu District, Changsha, 410008, China; e-mail: xyswcfh@csu.edu.cn

- Indicates open access to non-subscribers at www.ajnr.org

7 Indicates article with supplemental on-line photo.

http://dx.doi.org/10.3174/ajnr.A6615 divert blood flow from the direction of flow of the parent vessels are a risk factor for aneurysm formation. ${ }^{1,5,6}$ Previous studies have reported that the inclined MCA bifurcations with a widening angle are likely to harbor aneurysms, ${ }^{7,8}$ but mechanisms by which the aneurysms develop on MCA bifurcations are controversial and need to be elucidated. ${ }^{8-11}$ The present study was designed to extract the morphologic features of aneurysmal MCA bifurcations from clinical data, build a simplified MCA bifurcation model based on these extracted features, perform computational fluid dynamics (CFD) analysis on the aneurysmal MCA bifurcations and simplified models, and finally, to explore the hemodynamic mechanisms that could trigger aneurysm initiation.

\section{MATERIALS AND METHODS Case Selection and Matching}

Patients with unilateral MCA bifurcation aneurysms confirmed by CTA from July 2016 to March 2019 were enrolled in our study. Exclusion criteria were as follows: 1) fusiform or dissecting aneurysms, 2) trifurcation types of MCAs, and 3) aneurysms of $>10 \mathrm{~mm}$. In addition, we reviewed healthy subjects without 


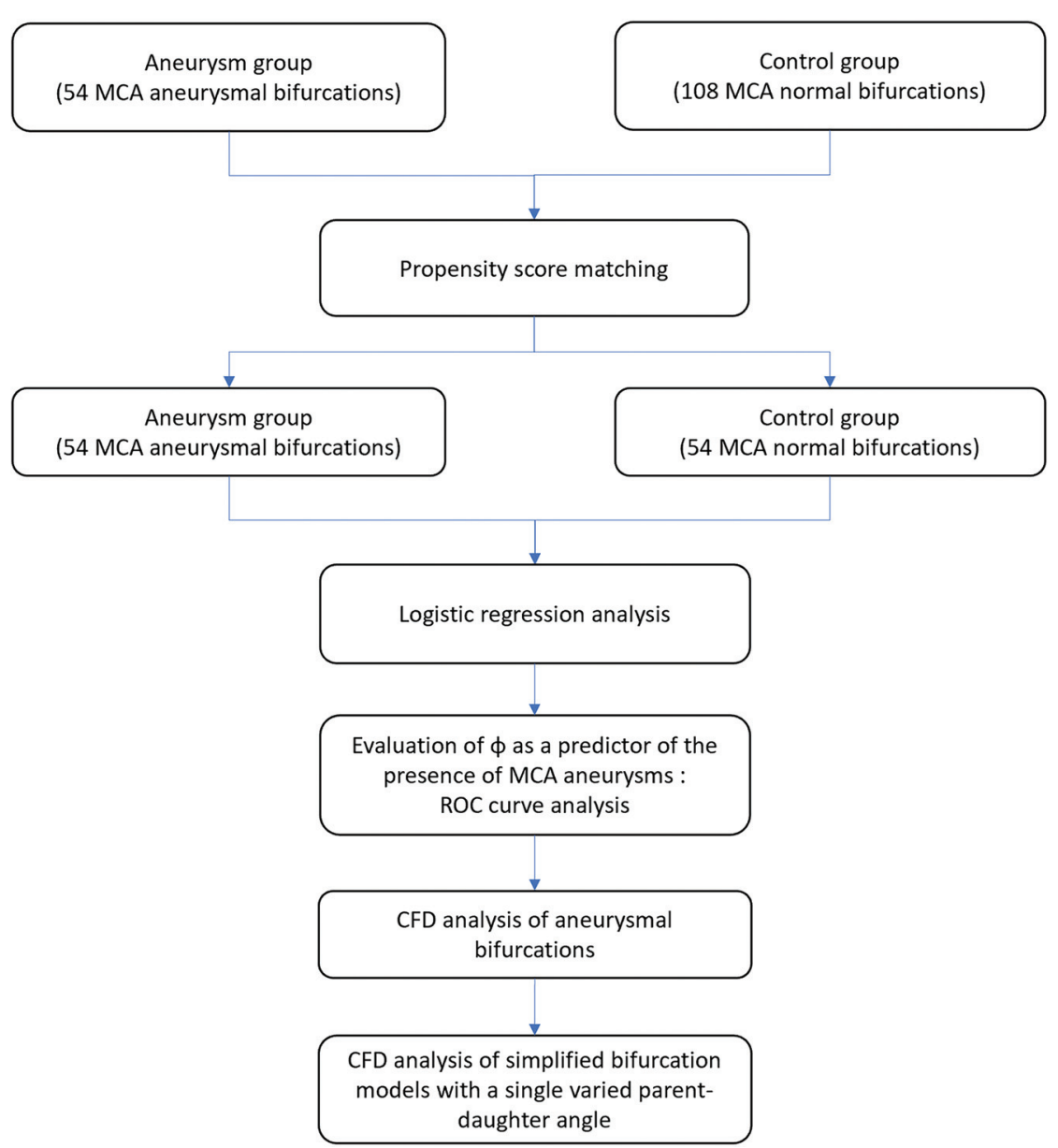

FIG 1. Flow diagram of the study population and procedure.
(F.Y.Z. and C.Q.C.), who were blinded to the clinical data, independently evaluated the morphologic data and resolved disagreements by consensus. The parent artery was defined as the M1 trunk of the MCA, and the daughter artery, as the M2 branch of the MCA.

The measuring methods of 6 angles in the control group are shown in Fig $2 A$. The measuring methods of diameters are depicted in Fig 2D. Four ratios of diameters (RDs) were used for analysis. They were separately defined as $\mathrm{RD}_{\mathrm{L} 1}$, the ratio of the left upper daughter-parent artery diameters; $\mathrm{RD}_{\mathrm{L} 2}$, the ratio of left lower daughter-parent artery diameters; $\mathrm{RD}_{\mathrm{R} 1}$, the ratio of right upper daughter-parent artery diameters; and $\mathrm{RD}_{\mathrm{R} 2}$, the ratio of right lower daughter-parent artery diameters.

The measuring methods of 6 angles in the aneurysm group are described in Fig $2 B$, and the diameter measuring methods are shown in Fig $2 D$. The ratio of parent-daughter artery diameters was separately termed as $\mathrm{RD}_{\mathrm{A}}$ on the aneurysm side and $\mathrm{RD}_{\mathrm{NA}}$ on the nonaneurysmal side. For the contralateral side, the ratio of upper daughter-parent artery diameters was defined as $\mathrm{RD}_{\mathrm{C} 1}$, and the ratio of lower daughter-parent artery diameters was $\mathrm{RD}_{\mathrm{C} 2}$. The method for deciding the location of an MCA bifurcation aneurysm is described in Fig $2 C^{12}$ The long axis of an aneurysm was measured as aneurysm size. Aneurysm neck width was also measured. with unilateral MCA aneurysms were assigned to the aneurysm group and 108 without intracranial aneurysms, to the control group. General data including age, sex, smoking, drinking, hypertension, hyperlipidemia, and diabetes were collected. Propensity score matching (PSM) was used for selecting the cases in the 2 groups, and the variables listed above were used as matching parameters. The matched cases included 54 with unilateral MCA aneurysms and 54 without intracranial aneurysms. A flow diagram of our study is shown in Fig 1. All procedures in this retrospective study that involved human participants were approved by the ethics committee of our hospital and were performed in accordance with the institutional ethics standards, the 1964 Declaration of Helsinki and its later amendments, or comparable ethical standards. Informed consent was obtained from all individual participants included in the study.

\section{Morphologic Features}

3D CTA data were reconstructed using Materialise Mimics software (Version 21.0; https://www.materialise.com/en/medical/mimicsinnovation-suite/mimics), and morphologic data were measured using the Materialise 3-matic research software (Version 13.0; https://www. materialise.com/en/software/3-matic). Two certified neuroradiologists

\section{CFD Analysis}

We reconstructed 3D MCA bifurcation models in Mimics research software (Materialise NV) for all 54 cases with MCA aneurysms and 20 cases in the control group. Then, the aneurysmal MCA bifurcation models were virtually removed using the 3-matic research software, and their initial status was simulated. All 3D models were exported to Fluent solver (Version 19.1; ANSYS) for CFD analysis.

After extracting some hemodynamic features from the above CFD analyses, we constructed a group of simplified 3D MCA bifurcations models with variable single parent-daughter angles. For these models, parent-daughter angle of the MCA bifurcations was set to $120^{\circ}$ (based on the mean value of $\phi_{\mathrm{NA}}$, $\left.118.81^{\circ} \pm 21.35^{\circ}\right)$, and another was varied from $120^{\circ}$ to $30^{\circ}$ with intervals of $10^{\circ}$ (based on the range of $\phi \mathrm{A}, 31.95^{\circ}-$ $\left.124.89^{\circ}\right)$. The diameters of the parent and daughter arteries were set to 4 and $3 \mathrm{~mm}$, respectively. For all 3D models, blood flow was modeled as a laminar Newtonian fluid, with a density of $1050 \mathrm{~kg} / \mathrm{m}^{3}$ and dynamic viscosity of $0.0032 \mathrm{~Pa} \times \mathrm{s}$. The inlet boundary of the parent artery was defined as the mass- 

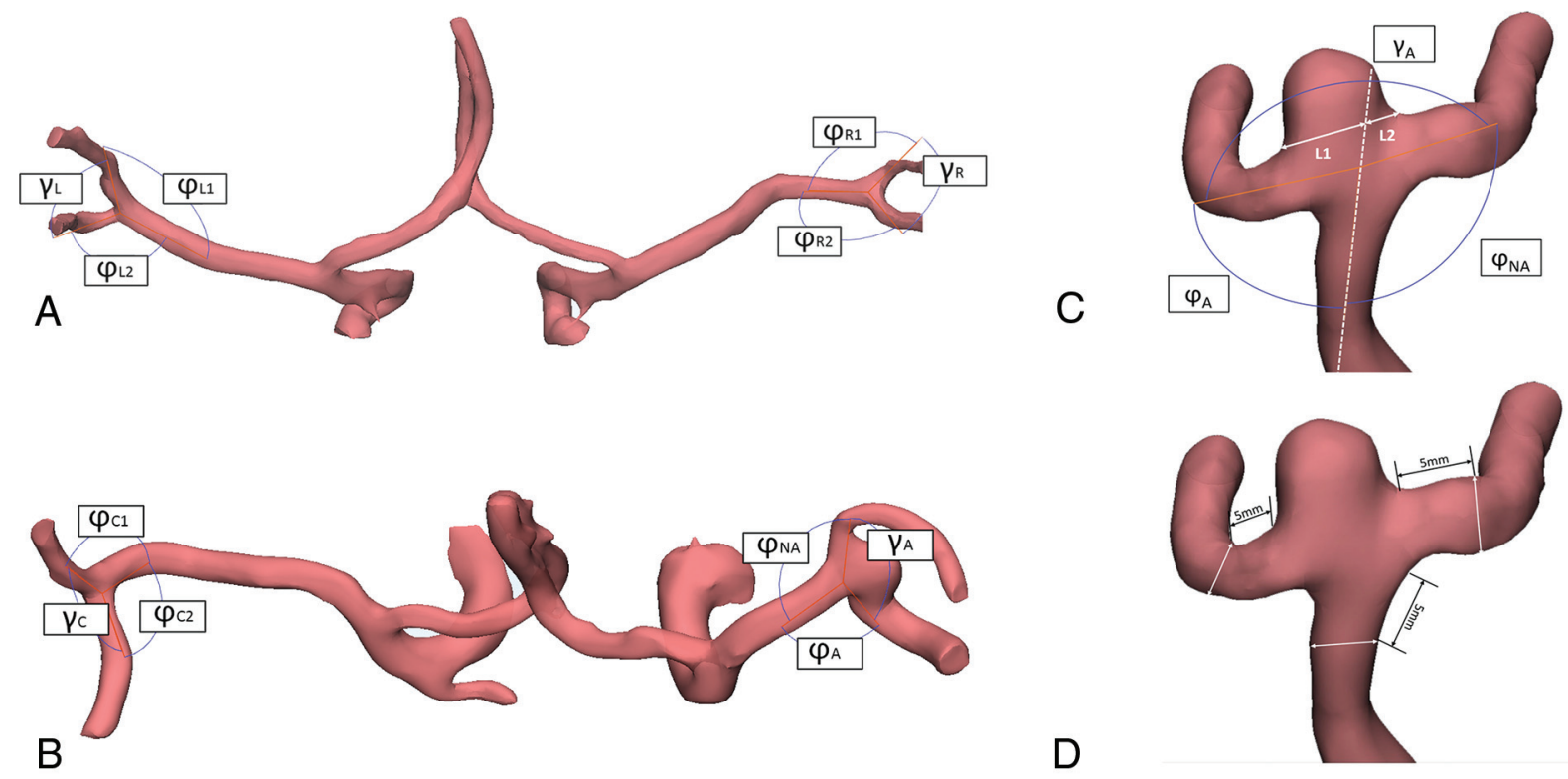

FIG 2. Schematic drawing of the angles measured in our study. $A$ and $B$, The parent-daughter angle was defined as the angle between the $M 1$ trunk and different M2 branches, and the daughter-daughter angle was the angle between $2 \mathrm{M} 2$ branches. $C$, In the MCA aneurysm bifurcation, lines $L 1$ and $L 2$ were crossed in the aneurysm neck and then measured to decide on which side of the M2 branch the aneurysm was located. $D$, The measurements of diameters were performed $5 \mathrm{~mm}$ beyond the bifurcation apex.

Table 1: Summary of clinical data

\begin{tabular}{|c|c|c|c|c|c|c|}
\hline & \multicolumn{3}{|c|}{ Before PSM } & \multicolumn{3}{|c|}{ After PSM ${ }^{a}$} \\
\hline & $\begin{array}{c}\text { Control Group } \\
(n=108)\end{array}$ & $\begin{array}{l}\text { Aneurysm Group } \\
(n=54)\end{array}$ & $P$ Value & $\begin{array}{l}\text { Control Group } \\
(n=54)\end{array}$ & $\begin{array}{c}\text { Aneurysm Group } \\
(n=54)\end{array}$ & $P$ Value \\
\hline Male sex (\%) & $61(56.5)$ & $18(33.3)$ & .009 & $26(48.1)$ & $18(33.3)$ & .17 \\
\hline Age (mean) (yr) & $43.65 \pm 19.34$ & $60.35 \pm 9.87$ & $<.001$ & $57.39 \pm 8.79$ & $60.35 \pm 9.87$ & .102 \\
\hline Hypertension (\%) & $30(27.8)$ & $29(53.7)$ & .002 & $25(46.3)$ & $29(53.7)$ & .564 \\
\hline Diabetes (\%) & 15 (13.9) & $8(14.8)$ & 1 & $12(22.2)$ & $8(14.8)$ & .457 \\
\hline Hyperlipidemia (\%) & $24(22.2)$ & $28(51.9)$ & $<.001$ & $19(35.2)$ & $28(51.9)$ & .12 \\
\hline Smoking (\%) & $30(27.8)$ & $12(22.2)$ & .568 & $20(37.0)$ & $12(22.2)$ & .14 \\
\hline Alcohol use (\%) & $27(25.0)$ & $9(16.7)^{\prime}$ & .316 & $16(29.6)$ & 9 (16.7) & .171 \\
\hline$\Phi$ (mean) & $124.88^{\circ} \pm 10.92^{\circ}$ & $107.98^{\circ} \pm 12.3^{\circ}$ & $<.001$ & $120.27^{\circ} \pm 10.50^{\circ}$ & $107.98^{\circ} \pm 12.31^{\circ}$ & $<.001$ \\
\hline$\gamma$ (mean) & $98.25^{\circ} \pm 20.54^{\circ}$ & $128.51^{\circ} \pm 16.69^{\circ}$ & $<.001$ & $106.68^{\circ} \pm 18.51^{\circ}$ & $128.51^{\circ} \pm 16.69^{\circ}$ & $<.001$ \\
\hline $\mathrm{RD}$ (mean) (mm) & $0.76 \pm 0.08$ & $0.79 \pm 0.11$ & .022 & $0.76 \pm 0.08$ & $0.79 \pm 0.11$ & .093 \\
\hline
\end{tabular}

${ }^{a}$ Variables as parameters for PSM included sex, age, hypertension, diabetes, hyperlipidemia, smoking, and Alcohol use (rows 1-7 in Table 1).

flow inlet at $0.0034 \mathrm{~kg} / \mathrm{s}$, and the outlet boundaries of 2 daughter arteries were defined as zero-static pressure. ${ }^{8,13,14}$

\section{Statistical Analysis}

Data were analyzed using R Studio (Version 1.1.383; http://rstudio. org/download/desktop). Student $t$ tests and ANOVA analyses were used to compare continuous variables, and the Pearson $\chi^{2}$ test was used for comparing categoric variables. Pearson correlation tests were used to analyze correlations between 2 continuous variables. Multiple variables were analyzed using logistic regression. To predict which daughter artery was prone to developing an MCA aneurysm, receiver operating characteristic (ROC) curve analysis was performed. $P$ values $<.05$ were considered statistically significant.

\section{RESULTS}

\section{Demographics}

The clinical data of both groups before and after PSM are summarized in Table 1. Of 54 subjects in the control group, 28 were women and 26 men, with a mean age of $57.39 \pm$ 8.79 years. Of 54 patients with aneurysms, 36 were women and 18 men, with a mean age of $60.35 \pm 9.87$ years. Twentytwo $(40.74 \%)$ patients were confirmed to have ruptured aneurysms, and 17 (31.48\%), to have multiple aneurysms. Logistic regression analysis based on morphologic parameters of MCA bifurcations showed that the presence of MCA aneurysms correlated with the parent-daughter angle (Table 2).

\section{Morphologic Features of Nonaneurysmal and Aneurysmal MCA Bifurcations}

There was no statistically significant difference between groups among the 7 parent-daughter angles $\left(\phi_{\mathrm{L} 1}, \phi_{\mathrm{L} 2}, \phi_{\mathrm{R} 1}, \phi_{\mathrm{R} 2}, \phi_{\mathrm{NA}}\right.$, $\phi_{\mathrm{C} 1}, \phi_{\mathrm{C} 2}$; Table 3 and Fig $3 A$ ). The daughter-daughter angles $\left(\gamma_{\mathrm{A}}\right.$ and $\left.\gamma_{\mathrm{C}}\right)$ in patients with aneurysms were significantly larger than those $\left(\gamma_{\mathrm{L}}\right.$ and $\left.\gamma_{\mathrm{R}}\right)$ in the control group (Table 3 and vFig $3 B$ ). 
For the aneurysm group, parent-daughter angles on the inclining side of the aneurysm $\left(\phi_{\mathrm{A}}\right)$ were significantly smaller than the other 3 parent-daughter angles $\left(\phi_{\mathrm{NA}}, \phi_{\mathrm{C} 1}, \phi_{\mathrm{C} 2}\right)$ (Fig $3 A$ ), whereas the daughter-daughter angles on the aneurysm side $\left(\gamma_{\mathrm{A}}\right)$ were significantly larger than the angle of the contralateral side $\left(\gamma_{\mathrm{C}}\right)($ Fig $3 B)$. The mean aneurysm size was $5.05 \pm 1.98 \mathrm{~mm}$, and the mean aneurysm neck diameter was $4.68 \pm 1.54 \mathrm{~mm}$. The optimal parent-daughter angle threshold to predict the daughter artery that was likely to harbor an MCA aneurysm was determined by ROC analysis. The ROC curve showed that the optimal threshold was 100.06 (area under the curve, 0.903), with $84.1 \%$ sensitivity and $81.5 \%$ specificity (Fig $3 C$ ).

\section{Influence of Aneurysm Rupture on MCA Bifurcation Morphology}

There was no significant difference between the parent-daughter and daughter-daughter angles or the RDs on aneurysmal and nonaneurysmal sides of the ruptured and unruptured aneurysmal MCA bifurcations (Table 4).

\section{Influence of Aneurysm Size and Neck Width on Parent- Daughter Angles of MCA Bifurcations}

Correlation analysis showed that the sizes and neck widths of MCA aneurysms were not significantly correlated with parentdaughter angles (On-line Figure).

\section{Hemodynamic Features of Aneurysmal MCA Bifurcations and Simplified MCA Bifurcation Models}

The CFD analysis performed on all MCA aneurysms showed that after the aneurysms were virtually removed, compared with control MCA bifurcations (On-line Digital Content 1; https:// yaleedu-my.sharepoint.com/:b:/g/personal/fenghua_chen_yale_ edu/EV5HuHzoJrRBsp1nO86pKgQBxPemPz4zSsXWBH2Ap$\mathrm{eR} 3 \mathrm{w} ? \mathrm{e}=\mathrm{u} 79 \mathrm{Us} 0$ ), their initial sites presented high-pressure

Table 2: Logistic regression analysis of morphologic factors associated with MCA aneurysms after PSM

\begin{tabular}{lcrc}
\hline & OR & \multicolumn{1}{c}{$95 \% \mathrm{CI}$} & $\boldsymbol{P}$ Value \\
\hline (Intercept) & 0.000 & $0.000-0.061$ & .006 \\
Mean parent-daughter angle & 1.095 & $1.053-1.145$ & $<.001$ \\
Mean RD & 0.130 & $0.001-15.004$ & .406 \\
\hline
\end{tabular}

regions (HPRs) and low-wall shear stress regions (LWSSRs) (Fig $4 \mathrm{~A}$ and On-line Digital Content 2; https://yaleedu-my. sharepoint.com/:b:/g/personal/fenghua_chen_yale_edu/ EV5HuHzoJrRBsp1nO86pKgQBxPemPz4zSsXWBH2Ap-eR3w?e= u79Us0). The CFD analysis of simplified 3D MCA bifurcation models revealed that when a single parent-daughter angle decreased from $120^{\circ}$ to $30^{\circ}$, the HPR and LWSSR shifted from the apex of the bifurcation to the daughter artery wall on the same side, and the maximum shifted distance was $2.03 \mathrm{~mm}$ (Fig $4 B-D$ and On-line Digital Content 3; https:// yaleedu-my.sharepoint.com/:b:/g/personal/fenghua_chen_yale_ edu/EV5HuHzoJrRBsp1nO86pKgQBxPemPz4zSsXWBH2Ap$\mathrm{eR} 3 \mathrm{w} ? \mathrm{e}=\mathrm{u} 79 \mathrm{Us} 0)$.

\section{DISCUSSION}

The mechanism of intracranial aneurysm initiation remains unclear. Intracranial arterial bifurcations frequently harbor aneurysms. When blood flow enters an arterial bifurcation, it exhibits complex hemodynamic features, some of which may correlate with aneurysm formation. ${ }^{2,5,15,16}$ In this study, we analyzed a series of MCA bifurcations with and without aneurysms and explored the hemodynamic factors involved in aneurysm development. We found that MCA aneurysms were commonly located on the inclined MCA bifurcations, consistent with previous studies. ${ }^{8,10}$ Our results revealed that inclined MCA aneurysmal bifurcations are usually caused by smaller single parent-daughter angles, but other parent-daughter angles maintain sizes similar to the 2 parent-daughter angles on the contralateral "normal" MCA bifurcations. CFD analysis based on the above morphologic features showed that when MCA bifurcations become inclined, the HPRs and LWSSRs shift from the apexes of the arterial bifurcations to the ipsilateral daughter arteries, and this hemodynamic feature may correlate with the initiation of MCA bifurcation aneurysms.

Considering that the parent-daughter angle may be affected by the aneurysm size, we enrolled only patients with small MCA aneurysms. We analyzed the correlation between the parent-daughter angle of the MCA bifurcation and aneurysm size and neck width. The results suggested that the size of the parent-daughter angle on the aneurysm side might not be affected by aneurysm growth. Our data also showed that aneurysm rupturing had little influence on MCA bifurcation morphology. ${ }^{8}$ Unlike some previous groups that based their measurements on $2 \mathrm{D}$ projecting images of MCA bifurcations, ${ }^{8,17}$ we used 3D measuring tools available in Mimics research software, so we did not adopt all the parameters used in those studies. We considered that by using the $3 \mathrm{D}$ coordinate, our data would be more representative of the real conditions in MCA bifurcations.

Some studies have reported that the daughter-daughter angle is correlated with the presence of an intracranial aneurysm, ${ }^{8,10}$ which is consistent with our results. We found that of all the parent-daughter angles in the aneurysm 

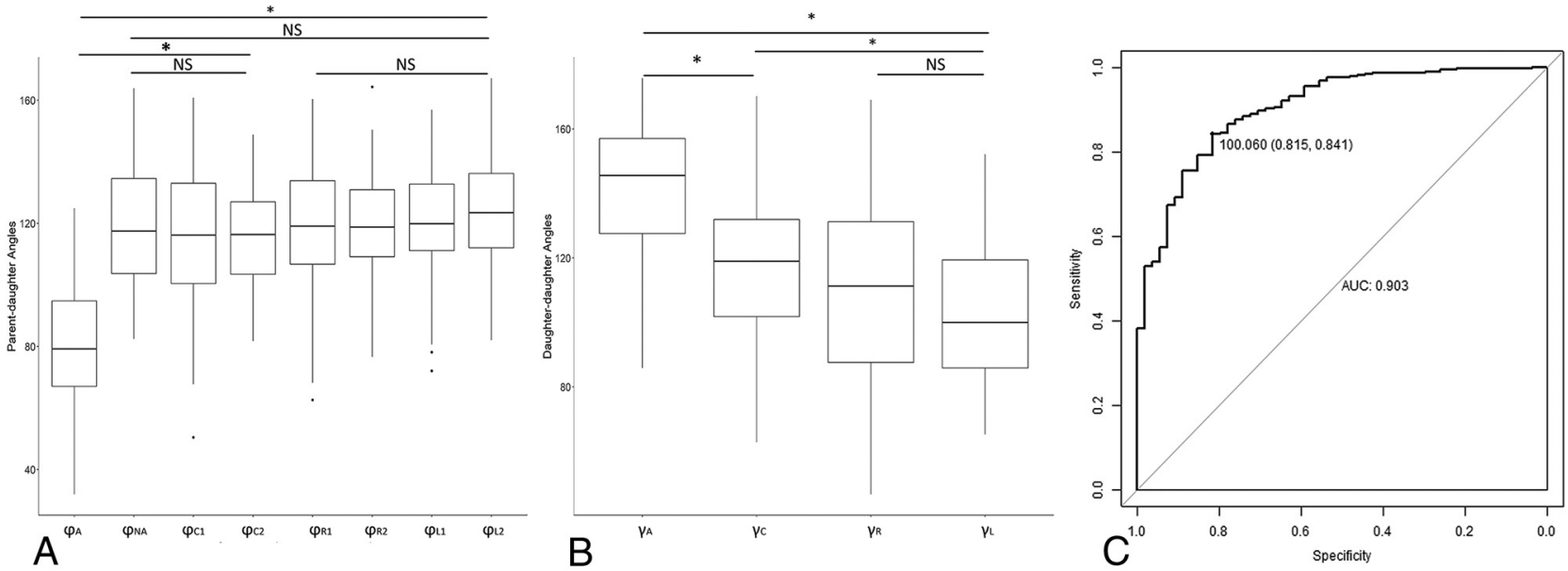

FIG 3. $A$, The size of $\phi \mathrm{A}$ was significantly different from that of the other 3 parent-daughter angles in the aneurysmal group and the 4 parentdaughter angles in the control group. There were no statistically significant differences among the 3 parent-daughter angles in the aneurysmal group and all 4 parent-daughter angles in the control group. B. The size of $\gamma_{\mathrm{A}}$ was significantly different from that of another daughter-daughter angle in the aneurysmal group and 2 daughter-daughter angles in the control group. There were no statistically significant differences between the 2 daughter-daughter angles in the control group. C, An optimal threshold of $100.06^{\circ}$ for $\phi \mathrm{A}$ (area under the curve, 0.903 ) with $84.1 \%$ sensitivity and $81.5 \%$ specificity revealed that the daughter artery that is apt to harbor an MCA aneurysm is the artery with a smaller parent-daughter angle. NS indicates no statistical significance $(P>.05)$; asterisk, statistical significance $(P<.05)$.

Table 4: Comparison of angles and diameters between ruptured and unruptured MCA aneurysms

\begin{tabular}{|c|c|c|c|c|}
\hline Variables & & Ruptured Aneurysm ${ }^{\mathrm{a}} n=22$ & Unruptured Aneurysm $^{\mathrm{a}} n=23$ & $P$ Value \\
\hline \multirow[t]{4}{*}{ Parent-daughter angles } & $\phi_{\mathrm{A}}\left(^{\circ}\right)$ & $84.08 \pm 20.77$ & $79.53 \pm 19.74$ & .423 \\
\hline & $\phi_{\mathrm{NA}}\left(^{\circ}\right)$ & $120.22 \pm 21.17$ & $117.85 \pm 21.76$ & .691 \\
\hline & $\phi_{\mathrm{Cl}}\left(^{\circ}\right)$ & $115.35 \pm 22.45$ & $117.15 \pm 23.52$ & .777 \\
\hline & $\phi_{\mathrm{C} 2}\left(^{\circ}\right)$ & $117.53 \pm 18.56$ & $113.78 \pm 16.43$ & .449 \\
\hline \multirow[t]{2}{*}{ Daughter-daughter angles } & $\gamma_{\mathrm{A}}\left(\left(^{\circ}\right)\right.$ & $137.01 \pm 24.69$ & $143.97 \pm 17.99$ & .265 \\
\hline & $\gamma_{C}\left(^{\circ}\right)$ & $117.67 \pm 19.04$ & $114.67 \pm 26.87$ & .634 \\
\hline \multirow[t]{2}{*}{ Aneurysmal side RDs } & $\mathrm{RD}_{\mathrm{A}}(\mathrm{mm})$ & $0.80 \pm 0.17$ & $0.73 \pm 0.22$ & .244 \\
\hline & $\mathrm{RD}_{\mathrm{NA}}(\mathrm{mm})$ & $0.90 \pm 0.22$ & $0.84 \pm 0.14$ & .234 \\
\hline \multirow[t]{2}{*}{ Nonaneurysmal side RDs } & $\mathrm{RD}_{\mathrm{Cl}}(\mathrm{mm})$ & $0.75 \pm 0.14$ & $0.72 \pm 0.20$ & .549 \\
\hline & $\mathrm{RD}_{\mathrm{C2}}(\mathrm{mm})$ & $0.78 \pm 0.14$ & $0.82 \pm 0.20$ & .469 \\
\hline
\end{tabular}

${ }^{a}$ Values are presented as means.

group, the only difference was observed in the parent-daughter angle $\left(\phi_{\mathrm{A}}\right)$ on the aneurysm inclining side. This suggests that the difference between the daughter-daughter angle $\left(\gamma_{\mathrm{A}}\right)$ on the aneurysmal side and the contralateral daughter-daughter angle $\left(\gamma_{\mathrm{c}}\right)$ is largely attributable to the parent-daughter angle $\left(\phi_{\mathrm{A}}\right)$. Moreover, ROC curve analysis showed that the parent-daughter angle $\left(\phi_{\mathrm{A}}\right)$ was useful for predicting which daughter artery is likely to harbor an MCA aneurysm. These results suggest that a single smaller parent-daughter angle may play an important role in MCA aneurysm occurrence. Prospective studies should be performed to test the predictive value in subjects with smaller parent-daughter angles of MCA bifurcations.

To explore the possible hemodynamic mechanism behind this unique shape, we further performed CFD analysis on $3 \mathrm{D}$ aneurysmal MCA bifurcations reconstructed from CTA data. By comparing the differences before and after virtual MCA aneurysm removal, we observed that the initiation sites of aneurysms overlapped with HPRs and LWSSRs, and these areas inclined to the daughter artery that had a smaller parent-daughter angle. Because hemodynamic data of CFD analysis on aneurysmal bifurcations are difficult to compare, we constructed a group of simplified bifurcation models to analyze hemodynamic changes on varying a single parent-daughter angle. The CFD analysis of these simplified models reconfirmed the previous finding in the $3 \mathrm{D}$ models of actual aneurysmal MCA bifurcations-that is, when one of the parent-daughter angles becomes smaller, the HPR and LWSSR shift from the apex of the arterial bifurcation to the daughter artery. We also observed that the HPR range widened and the distal high-pressure gradient became steeper, but the pressure and wall shear stress were not dramatically altered. Combining these results with the fact that the inclined HPR and LWSSR overlap with an MCA aneurysm site, we inferred that the inclined HPRs and LWSSRs may correlate with the initiation of the aneurysm.

According to previous studies, high or low wall shear stress (LWSS) may cause aneurysm formation. ${ }^{1,3,4,18}$ The wall shear stress curve data supported the hypothesis that LWSS was a possible reason. Similar LWSS values also exist on the apexes of normal MCA bifurcations, however, the normal distribution of LWSS seldom causes aneurysms. Rather, the abnormal shift of LWSS may be the real reason. Why can the HPR and LWSSR on 


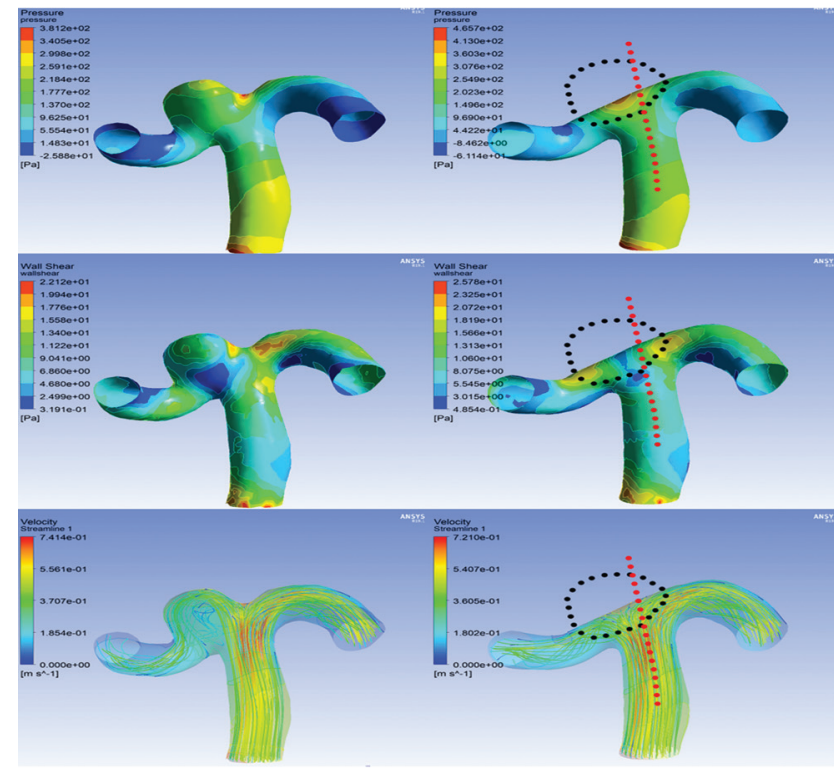

A

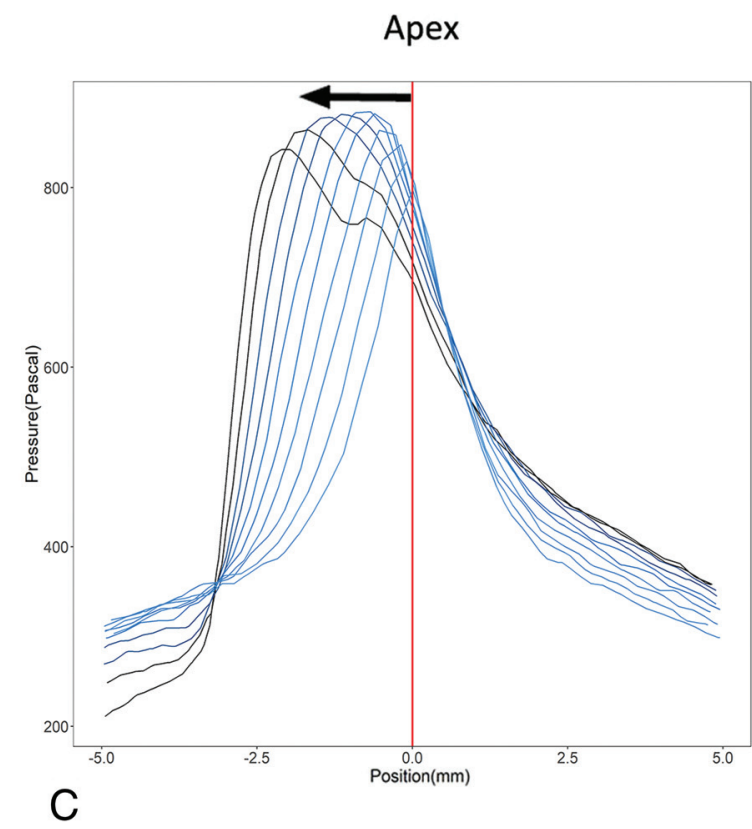

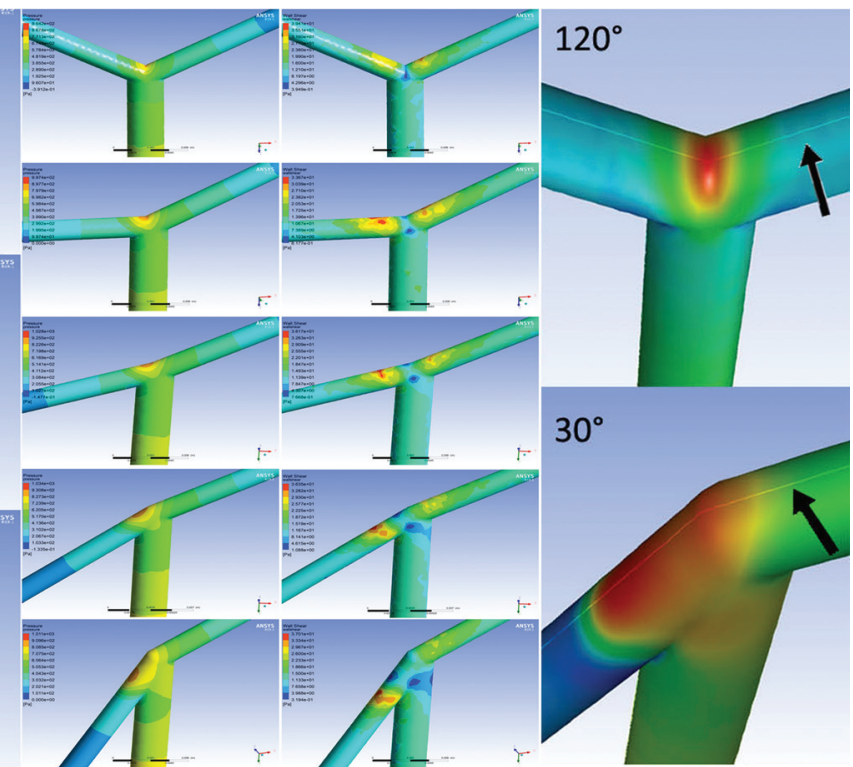

$\mathrm{B}$

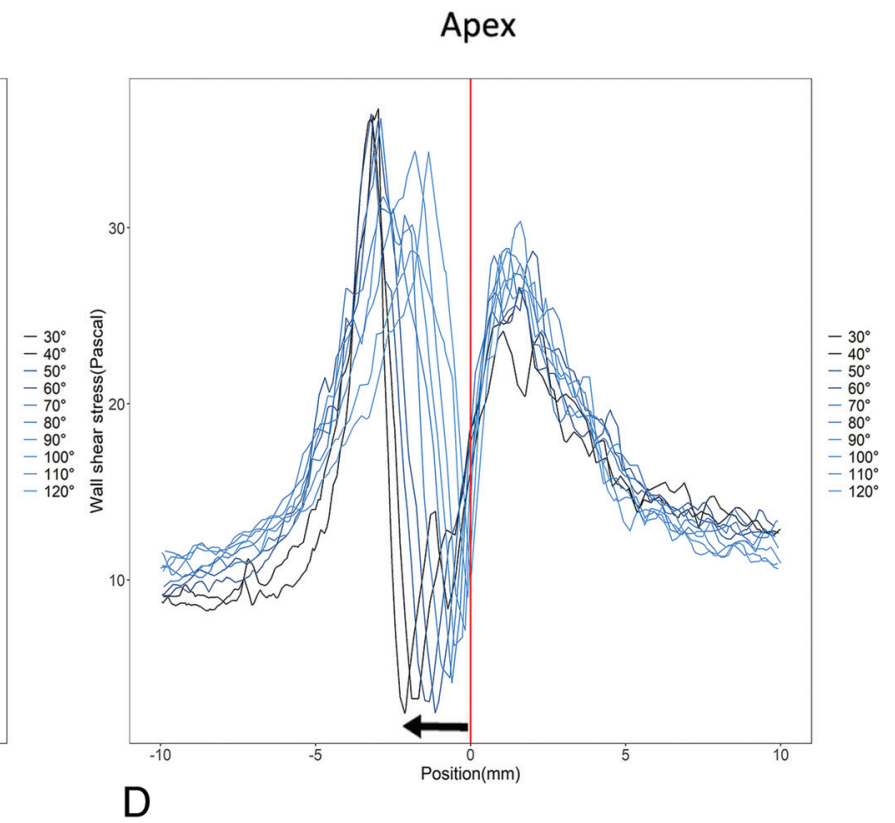

FIG 4. CFD analysis. A, CFD analysis on an aneurysmal MCA bifurcation shows the initial sites of aneurysms overlap with HPRs and LWSSRs, and these sites incline to the daughter artery with a smaller parent-daughter angle (first row, pressure contour; second row, wall shear stress contour; third row, blood flow direction. See also On-line Digital Content 2). B, CFD analysis of simplified 3D MCA bifurcation models. When a single parent-daughter angle varied from $120^{\circ}$ to $30^{\circ}$, HPRs and LWSSRs were displaced from the apex of the bifurcation to the same daughter artery wall (detailed demonstrations are also shown in On-line Digital Content 3); the values of hemodynamic parameters were obtained along the virtual path (black arrows) in the simplified 3D MCA bifurcation models. $C$ and $D$, The distribution of pressure and wall shear stress shifts along the virtual path when a single parent-daughter angle varies from $120^{\circ}$ to $30^{\circ}$. The HPR and LWSSR (black arrows) shift from the apex to the left daughter artery, which has a smaller parent-daughter angle. The red line represents the bifurcation apex, the left part represents the daughter artery with a variable angle, and the right part represents the daughter artery with a fixed angle.

the daughter arterial wall form an aneurysm but cannot when they are located on the apex? Previous studies offer some clues. ${ }^{3,18-22}$ Specifically, unlike the arterial wall, the apex of a cerebral arterial bifurcation lacks a tunica media and forms a "gap" that is filled with fibers from the tunica adventitia, namely the "apical ridge." This structure is composed of collagen fibers and can, therefore, bear stronger impingement of blood flow than the arterial wall.
On the basis of the above inferences, we propose the following mechanism of MCA aneurysm initiation. Compared with symmetric MCA bifurcations in which high-pressure and LWSS fall on the "strong" apical ridge, the high-pressure and LWSS of asymmetric MCA bifurcations fall on the relatively "weak" arterial wall. Because the arterial wall cannot bear this stress, arterial structures-especially the elastic laminas—will be damaged under the long-term impact of blood flow and an aneurysm will emerge 


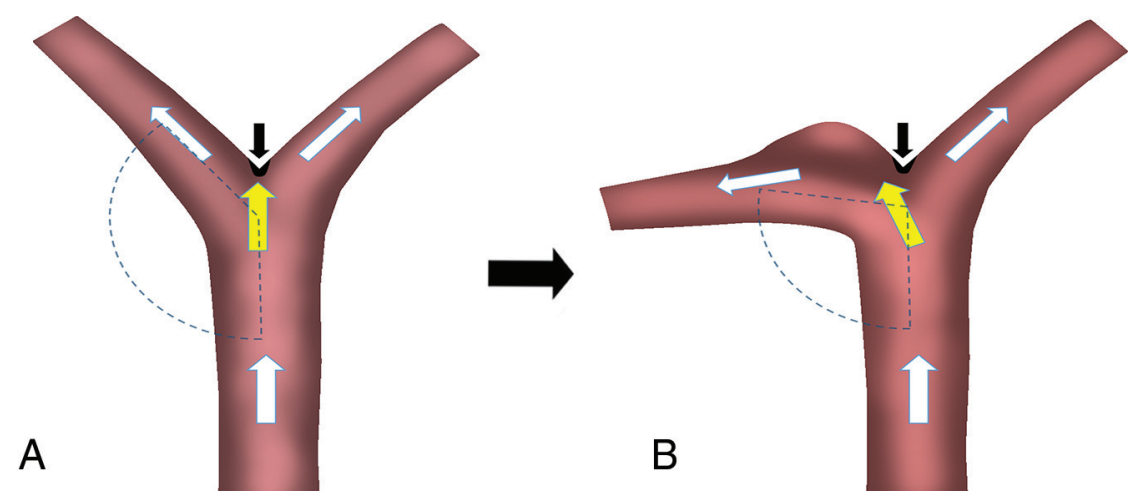

FIG 5. Schematic drawing of the hypothetic correlation between the inclined MCA bifurcation and aneurysm initiation. If the HPR and LWSSR locate on the strong apical ridge (black arrow), an MCA bifurcation with normal parent-daughter angles is not likely to develop an aneurysm $(A)$. If the HPR and LWSSR shift to the weak ipsilateral arterial wall (yellow arrow), an inclined MCA bifurcation with asymmetric parent-daughter angles is more likely to develop an aneurysm (B). White arrows indicate blood flow direction.

(Fig 5). Although this hypothesis considers the influence of the MCA bifurcation structure on the formation of an intracranial aneurysm to some extent, it also emphasizes the core role of hemodynamics. Whether these events are universal is unknown, and our theory still needs to be examined and confirmed by further investigations based on biologic models.

Our study has some limitations that need to be considered. First, we examined cross-sectional data. Although our analysis showed no significant correlation between parent-daughter angles on the aneurysmal bifurcations and aneurysm size or neck width, the local geometry of the arteries may still be affected, which would reduce the accuracy of hemodynamic analysis. To minimize the effect of aneurysm size and represent the initial status of aneurysm formation, only small MCA aneurysms of $<10 \mathrm{~mm}$ were included in our study; therefore, our results may not be generalizable to all MCA aneurysms. Second, our findings revealed a stronger correlation between the parent-daughter angle of the MCA bifurcation and the presence of aneurysms; however, it is unclear whether this is a cause-and-effect relationship. Future studies should be performed to help identify high-risk individuals who are likely to develop aneurysms.

\section{CONCLUSIONS}

An inclined MCA bifurcation caused by a single smaller parent-daughter angle is prone to develop an aneurysm. When a single parent-daughter angle of an MCA bifurcation decreases, the HPR and LWSSR shift from the apex of the arterial bifurcation to the daughter artery wall. This hemodynamic change may be associated with the initiation of an MCA bifurcation aneurysm.

\section{ACKNOWLEDGMENTS}

The authors thank Jun Zhou, $\mathrm{PhD}$ (College of Chemistry and Chemical Engineering, Hunan University), for assistance with CFD analysis.
Disclosures: Zheng Huang-RELATED: Grant: National Natural Science Foundation of China (grant No. 81873756), Comments: The grant was used to buy some software for this study.* Feiyue Y. ZengRELATED: Grant. National Science Foundation of China (grant No.81873756).* Longbo B. ZhangRELATED: Grant: National Natural Science Foundation of China (grant No.81873756).* Fenghua H. Chen-RELATED: Grant: National Natural Science Foundation of China (grant No. 81873756).* *Money paid to the institution

\section{REFERENCES}

1. Kulcsár Z, Ugron Á, Marosfoi M, et al. Hemodynamics of cerebral aneurysm initiation: the role of wall shear stress and spatial wall shear stress gradient. AJNR Am J Neuroradiol 2011;32:587-94 CrossRef Medline

2. Wang J, Tan HQ, Zhu YQ, et al. Complex hemodynamic insult in combination with wall degeneration at the apex of an arterial bifurcation contributes to generation of nascent aneurysms in a canine model. $A J N R$ Am J Neuroradiol 2014;35:1805-12 CrossRef Medline

3. Xiao W, Qi T, He S, et al. Low wall shear stress is associated with local aneurysm wall enhancement on high-resolution MR vessel wall imaging. AJNR Am J Neuroradiol 2018;39:2082-87 CrossRef

4. Diagbouga MR, Morel S, Bijlenga $P$, et al. Role of hemodynamics in initiation/growth of intracranial aneurysms. Eur J Clin Invest 2018;48:e12992 CrossRef

5. Meng H, Wang Z, Hoi Y, et al. Complex hemodynamics at the apex of an arterial bifurcation induces vascular remodeling resembling cerebral aneurysm initiation. Stroke 2007;38:1924-31 CrossRef Medline

6. Chien A, Sayre J. Morphologic and hemodynamic risk factors in ruptured aneurysms imaged before and after rupture. AJNR Am J Neuroradiol 2014;35:2130-35 CrossRef Medline

7. Sadatomo T, Yuki K, Migita K, et al. Differences between middle cerebral artery bifurcations with normal anatomy and those with aneurysms. Neurosurg Rev 2013;36:437-45 CrossRef Medline

8. Song J, Zhu F, Qian Y, et al. Morphological and hemodynamic differences between aneurysmal middle cerebral artery bifurcation and contralateral nonaneurysmal anatomy. Neurosurgery 2017;81:779-86 CrossRef Medline

9. Can A, Ho AL, Dammers R, et al. Morphological parameters associated with middle cerebral artery aneurysms. Neurosurgery 2015;76:721-26 CrossRef Medline

10. Baharoglu MI, Lauric A, Safain MG, et al. Widening and high inclination of the middle cerebral artery bifurcation are associated with presence of aneurysms. Stroke 2014;45:2649-55 CrossRef Medline

11. Farnoush A, Avolio A, Qian Y. Effect of bifurcation angle configuration and ratio of daughter diameters on hemodynamics of bifurcation aneurysms. AJNR Am J Neuroradiol 2013;34:391-96 CrossRef Medline

12. Zhang XJ, Gao BL, Li TX, et al. Association of basilar bifurcation aneurysms with age, sex, and bifurcation geometry. Stroke 2018;49:1371-76 CrossRef

13. Jansen IG, Schneiders JJ, Potters WV, et al. Generalized versus patient-specific inflow boundary conditions in computational fluid dynamics simulations of cerebral aneurysmal hemodynamics. AJNR Am J Neuroradiol 2014;35:1543-48 CrossRef Medline

14. Karmonik C. Toward improving fidelity of computational fluid dynamics simulations: Boundary conditions matter. AJNR Am J Neuroradiol 2014;35:1549-50 CrossRef Medline

15. Alfano JM, Kolega J, Natarajan SK, et al. Intracranial aneurysms occur more frequently at bifurcation sites that typically experience 
higher hemodynamic stresses. Neurosurgery 2013;73:497-505 CrossRef Medline

16. Chung BJ, Mut F, Putman CM, et al. Identification of hostile hemodynamics and geometries of cerebral aneurysms: a case-control study. AJNR Am J Neuroradiol 2018;39:1860-66 CrossRef Medline

17. Tütüncü F, Schimansky S, Baharoglu MI, et al. Widening of the basilar bifurcation angle: association with presence of intracranial aneurysm, age, and female sex. J Neurosurg 2014;121:1401-10 CrossRef Medline

18. Meng H, Tutino VM, Xiang J, et al. High WSS or Low WSS? Complex interactions of hemodynamics with intracranial aneurysm initiation, growth, and rupture: toward a unifying hypothesis. AJNR Am J Neuroradiol 2014;35:1254-62 CrossRef Medline
19. Rowe AJ, Finlay HM, Canham PB. Collagen biomechanics in cerebral arteries and bifurcations assessed by polarizing microscopy. $J$ Vasc Res 2003;40:406-15 CrossRef Medline

20. Yamazoe N, Hashimoto N, Kikuchi H, et al. Elastic skeleton of intracranial cerebral aneurysms in rats. Stroke 1990;21:1722-26 CrossRef Medline

21. Kim C, Kikuchi H, Hashimoto N, et al. Involvement of internal elastic lamina in development of induced cerebral aneurysms in rats. Stroke 1988;19:507-11 CrossRef Medline

22. Cebral JR, Duan X, Chung BJ, et al. Wall mechanical properties and hemodynamics of unruptured intracranial aneurysms. AJNR Am J Neuroradiol 2015;36:1695-703 CrossRef Medline 